\title{
Approximation with an arbitrary order by generalized Kantorovich-type and Durrmeyer-type operators on $[0,+\infty)$
}

\author{
Sorin Trifa
}

\begin{abstract}
Given an arbitrary sequence $\lambda_{n}>0, n \in \mathbb{N}$, with the property that $\lim _{n \rightarrow \infty} \lambda_{n}=0$ as fast we want, in this note we introduce modified/ generalized Szász-Kantorovich, Baskakov-Kantorovich, Szász-Durrmeyer-Stancu and Baskakov-Szász-Durrmeyer-Stancu operators in such a way that on each compact subinterval in $[0,+\infty)$ the order of uniform approximation is $\omega_{1}\left(f ; \sqrt{\lambda_{n}}\right)$. These modified operators uniformly approximate a Lipschitz 1 function, on each compact subinterval of $[0, \infty)$ with the arbitrary good order of approximation $\sqrt{\lambda_{n}}$. The results obtained are of a definitive character (that is are the best possible) and also have a strong unifying character, in the sense that for various choices of the nodes $\lambda_{n}$, one can recapture previous approximation results obtained for these operators by other authors.
\end{abstract}

Mathematics Subject Classification (2010): 41A36, 41A25.

Keywords: Generalized Szász-Kantorovich operators, generalized BaskakovKantorovich operators, generalized Szász-Durrmeyer-Stancu operators, generalized Baskakov-Szász-Durrmeyer-Stancu operators, linear and positive operators, modulus of continuity, arbitrary order of approximation.

\section{Introduction}

It is known that the classical Baskakov operators are given by the formula (see, e.g., $[2])$

$$
\begin{aligned}
V_{n}(f)(x) & =\sum_{j=0}^{\infty}\left(\begin{array}{c}
n+j-1 \\
j
\end{array}\right) \frac{x^{j}}{(1+x)^{n+j}} f\left(\frac{j}{n}\right)=(1+x)^{-n} \sum_{j=0}^{\infty} \frac{(n+j-1) !}{j !(n-1) !} \frac{x^{j}}{(1+x)^{j}} \\
& =(1+x)^{-n} \sum_{j=0}^{\infty} \frac{n(n+1) \ldots(n+j-1)}{j !} \frac{x^{j}}{(1+x)^{j}} .
\end{aligned}
$$


In the recent paper [9], this operator was modified by replacing $n$ with $\frac{1}{\lambda_{n}}$, where $\lim _{n \rightarrow \infty} \lambda_{n}=0$ as fast we want, and the approximation properties (of arbitrary good order depending on $\lambda_{n}$ ) of the new obtained Baskakov operator defined by the formula $V_{n}\left(f ; \lambda_{n}\right)(x)=(1+x)^{\frac{-1}{\lambda_{n}}} \sum_{j=0}^{\infty} \frac{1}{j !} \frac{1}{\lambda_{n}}\left(1+\frac{1}{\lambda_{n}}\right) \ldots\left(j-1+\frac{1}{\lambda_{n}}\right)\left(\frac{x}{1+x}\right)^{j} f\left(j \lambda_{n}\right), x \geq 0$,

were obtained. Above by convention,

$$
\frac{1}{j !} \frac{1}{\lambda_{n}}\left(1+\frac{1}{\lambda_{n}}\right) \ldots\left(j-1+\frac{1}{\lambda_{n}}\right)=1 \text { for } j=0 .
$$

The complex variable case for $V_{n}\left(f ; \lambda_{n}\right)$ was studied in [10]. Also, in [6], the above idea was applied to the Jakimovski-Leviatan-Ismail kind generalization of Szász-Mirakjan operators.

The goal of the present paper is that based on the above idea, to introduce modified/generalized Szász-Kantorovich, Baskakov-Kantorovich, Szász-Durrmeyer-Stancu and Baskakov-Szász-Durrmeyer-Stancu operators in such a way that on each compact subinterval in $[0,+\infty)$ the order of uniform approximation is $\omega_{1}\left(f ; \sqrt{\lambda_{n}}\right)$. These modified operators can uniformly approximate a Lipschitz 1 function, on each compact subinterval of $[0, \infty)$ with the arbitrary good order of approximation $\sqrt{\lambda_{n}}$ given at the beginning.

In conclusion, it is worth mentioning for these generalized operators that since $\lambda_{n}$ ca be chosen with $\lambda_{n} \searrow 0$ arbitrary fast, in fact it follows that the order of convergence $\omega_{1}\left(f ; \sqrt{\lambda_{n}}\right)$ is arbitrary good. For this reason, the results obtained by this paper have a definitive character (that is they are the best possible). In the same time, the results also have a strong unifying character, in the sense that for various choices of the nodes $\lambda_{n}$ one can recapture previous approximation results obtained by other authors.

\section{Generalized Baskakov-Kantorovich operators}

In this section we deal with the Baskakov-Kantorovich operators.

It is known that the classical Baskakov-Kantorovich operators are defined by (see, e.g., [3])

$$
\begin{aligned}
K_{n}(f)(x) & =\sum_{j=0}^{\infty}\left(\begin{array}{c}
n+j-1 \\
j
\end{array}\right) \frac{x^{j}}{(1+x)^{n+j}} n \int_{j / n}^{(j+1) / n} f(v) d v \\
& =(1+x)^{-n} \sum_{j=0}^{\infty} \frac{n(n+1) \ldots(n+j-1)}{j !} \frac{x^{j}}{(1+x)^{j}} n \int_{j / n}^{(j+1) / n} f(v) d v
\end{aligned}
$$

If we replace $n$ with $\frac{1}{\lambda_{n}}$, then we obtain the generalized Baskakov-Kantorovich operators, defined by the formula

$$
\begin{aligned}
& K_{n}\left(f ; \lambda_{n}\right)(x) \\
& =(1+x)^{-\frac{1}{\lambda_{n}}} \sum_{j=0}^{\infty} \frac{1}{j !} \frac{1}{\lambda_{n}}\left(1+\frac{1}{\lambda_{n}}\right) \ldots\left(j-1+\frac{1}{\lambda_{n}}\right) \frac{x^{j}}{(1+x)^{j}} \frac{1}{\lambda_{n}} \int_{j \lambda_{n}}^{(j+1) \lambda_{n}} f(v) d v .
\end{aligned}
$$


Denote everywhere in the paper $e_{k}(x)=x^{k}, k=0,1,2, \ldots$

This section deals with the approximation properties of the operator $K_{n}\left(f ; \lambda_{n}\right)(x)$. For our purpose, firstly we need the following auxiliary result.

Lemma 2.1. We have:

(i) $K_{n}\left(e_{0} ; \lambda_{n}\right)(x)=1 ; K_{n}\left(e_{1} ; \lambda_{n}\right)(x)=x+\frac{1}{2} \cdot \lambda_{n}$;

$$
K_{n}\left(e_{2} ; \lambda_{n}\right)(x)=x^{2}+2 \lambda_{n} x+\lambda_{n} x^{2}+\frac{1}{3} \cdot \lambda_{n}^{2} ;
$$

(ii) $K_{n}\left((t-x)^{2} ; \lambda_{n}\right)(x)=\lambda_{n}\left(x^{2}+x+\frac{1}{3} \cdot \lambda_{n}\right)$.

Proof. By using the formulas in Corollary 2.1 in [9],

$$
V_{n}\left(e_{0} ; \lambda_{n}\right)(x)=1, \quad V_{n}\left(e_{1} ; \lambda_{n}\right)(x)=x
$$

and

$$
V_{n}\left(e_{2} ; \lambda_{n}\right)(x)=x^{2}+\lambda_{n} x(1+x)
$$

we will calculate $K_{n}\left(e_{0} ; \lambda_{n}\right)(x), K_{n}\left(e_{1} ; \lambda_{n}\right)(x), K_{n}\left(e_{2} ; \lambda_{n}\right)(x), K_{n}\left((t-x)^{2} ; \lambda_{n}\right)(x)$.

(i) Therefore,

$$
\begin{gathered}
K_{n}\left(e_{0} ; \lambda_{n}\right)(x) \\
=(1+x)^{-\frac{1}{\lambda_{n}}} \sum_{j=0}^{\infty} \frac{1}{j !} \frac{1}{\lambda_{n}}\left(1+\frac{1}{\lambda_{n}}\right) \ldots\left(j-1+\frac{1}{\lambda_{n}}\right)\left(\frac{x}{1+x}\right)^{j} \frac{1}{\lambda_{n}} \int_{j \lambda_{n}}^{(j+1) \lambda_{n}} d v \\
=(1+x)^{-\frac{1}{\lambda_{n}}} \sum_{j=0}^{\infty} \frac{1}{j !} \frac{1}{\lambda_{n}}\left(1+\frac{1}{\lambda_{n}}\right) \ldots\left(j-1+\frac{1}{\lambda_{n}}\right)\left(\frac{x}{1+x}\right)^{j} \frac{1}{\lambda_{n}}\left(j \lambda_{n}+\lambda_{n}-j \lambda_{n}\right) \\
=(1+x)^{-\frac{1}{\lambda_{n}}} \sum_{j=0}^{\infty} \frac{1}{j !} \frac{1}{\lambda_{n}}\left(1+\frac{1}{\lambda_{n}}\right) \ldots\left(j-1+\frac{1}{\lambda_{n}}\right)\left(\frac{x}{1+x}\right)^{j}=V_{n}\left(e_{0} ; \lambda_{n}\right)(x)=1 .
\end{gathered}
$$

Also,

$$
\begin{gathered}
=(1+x)^{-\frac{1}{\lambda_{n}}} \sum_{j=0}^{\infty} \frac{1}{j !} \frac{1}{\lambda_{n}}\left(1+\frac{1}{\lambda_{n}}\right) \ldots\left(j-1+\frac{1}{\lambda_{n}}\right)\left(\frac{x}{1+x}\right)^{j} \frac{1}{\lambda_{n}} \int_{j \lambda_{n}}^{(j+1) \lambda_{n}} v d v \\
\left.=(1+x)^{-\frac{1}{\lambda_{n}}}\right)(x) \\
\cdot \sum_{j=0}^{\infty} \frac{1}{j ! \lambda_{n}}\left(1+\frac{1}{\lambda_{n}}\right) \ldots\left(j-1+\frac{1}{\lambda_{n}}\right)\left(\frac{x}{1+x}\right)^{j} \frac{1}{2 \lambda_{n}}\left(j^{2} \lambda_{n}^{2}+\lambda_{n}^{2}+2 j \lambda_{n}^{2}-j^{2} \lambda_{n}^{2}\right) \\
=(1+x)^{-\frac{1}{\lambda_{n}}} \sum_{j=0}^{\infty} \frac{1}{j !} \frac{1}{\lambda_{n}}\left(1+\frac{1}{\lambda_{n}}\right) \ldots\left(j-1+\frac{1}{\lambda_{n}}\right)\left(\frac{x}{1+x}\right)^{j} \frac{1}{\lambda_{n}}\left(\frac{1}{2} \lambda_{n}^{2}+j \lambda_{n}^{2}\right) \\
=(1+x)^{-\frac{1}{\lambda_{n}}} \sum_{j=0}^{\infty} \frac{1}{j !} \frac{1}{\lambda_{n}}\left(1+\frac{1}{\lambda_{n}}\right) \ldots\left(j-1+\frac{1}{\lambda_{n}}\right)\left(\frac{x}{1+x}\right)^{j} \frac{1}{\lambda_{n}} \frac{1}{2} \lambda_{n}^{2} \\
+(1+x)^{-\frac{1}{\lambda_{n}}} \sum_{j=0}^{\infty} \frac{1}{j !} \frac{1}{\lambda_{n}}\left(1+\frac{1}{\lambda_{n}}\right) \ldots\left(j-1+\frac{1}{\lambda_{n}}\right)\left(\frac{x}{1+x}\right)^{j} \frac{1}{\lambda_{n}} j \lambda_{n}^{2} \\
=V_{n}\left(e_{1} ; \lambda_{n}\right)(x)+\frac{1}{2} \lambda_{n}(1+x)^{-\frac{1}{\lambda_{n}}} \sum_{j=0}^{\infty} \frac{1}{j !} \frac{1}{\lambda_{n}}\left(1+\frac{1}{\lambda_{n}}\right) \ldots\left(j-1+\frac{1}{\lambda_{n}}\right)\left(\frac{x}{1+x}\right)^{j}
\end{gathered}
$$


Then,

$$
=V_{n}\left(e_{1} ; \lambda_{n}\right)(x)+\frac{1}{2} \lambda_{n} V_{n}\left(e_{0} ; \lambda_{n}\right)(x)=x+\frac{1}{2} \lambda_{n} .
$$

$$
\begin{aligned}
& K_{n}\left(e_{2} ; \lambda_{n}\right)(x) \\
& =(1+x)^{-\frac{1}{\lambda_{n}}} \sum_{j=0}^{\infty} \frac{1}{j !} \frac{1}{\lambda_{n}}\left(1+\frac{1}{\lambda_{n}}\right) \ldots\left(j-1+\frac{1}{\lambda_{n}}\right)\left(\frac{x}{1+x}\right)^{j} \frac{1}{\lambda_{n}} \int_{j \lambda_{n}}^{(j+1) \lambda_{n}} v^{2} d v \\
& =(1+x)^{-\frac{1}{\lambda_{n}}} \sum_{j=0}^{\infty} \frac{1}{j !} \frac{1}{\lambda_{n}}\left(1+\frac{1}{\lambda_{n}}\right) \ldots\left(j-1+\frac{1}{\lambda_{n}}\right)\left(\frac{x}{1+x}\right)^{j} \frac{1}{\lambda_{n}} \frac{1}{3}\left((j+1)^{3} \lambda_{n}^{3}-j^{3} \lambda_{n}^{3}\right) \\
& =(1+x)^{-\frac{1}{\lambda_{n}}} \\
& \cdot \sum_{j=0}^{\infty} \frac{1}{j !} \frac{1}{\lambda_{n}}\left(1+\frac{1}{\lambda_{n}}\right) \ldots\left(j-1+\frac{1}{\lambda_{n}}\right)\left(\frac{x}{1+x}\right)^{j} \frac{1}{\lambda_{n}} \frac{1}{3}\left(j^{3} \lambda_{n}^{3}+3 j^{2} \lambda_{n}^{3}+3 j \lambda_{n}^{3}+\lambda_{n}^{3}-j^{3} \lambda_{n}^{3}\right) \\
& =(1+x)^{-\frac{1}{\lambda_{n}}} \sum_{j=0}^{\infty} \frac{1}{j !} \frac{1}{\lambda_{n}}\left(1+\frac{1}{\lambda_{n}}\right) \ldots\left(j-1+\frac{1}{\lambda_{n}}\right)\left(\frac{x}{1+x}\right)^{j} \frac{1}{\lambda_{n}}\left(j^{2} \lambda_{n}^{3}+j \lambda_{n}^{3}+\frac{1}{3} \lambda_{n}^{3}\right) \\
& =(1+x)^{-\frac{1}{\lambda_{n}}} \sum_{j=0}^{\infty} \frac{1}{j !} \frac{1}{\lambda_{n}}\left(1+\frac{1}{\lambda_{n}}\right) \ldots\left(j-1+\frac{1}{\lambda_{n}}\right)\left(\frac{x}{1+x}\right)^{j} \frac{1}{\lambda_{n}} j^{2} \lambda_{n}^{3} \\
& +(1+x)^{-\frac{1}{\lambda_{n}}} \sum_{j=0}^{\infty} \frac{1}{j !} \frac{1}{\lambda_{n}}\left(1+\frac{1}{\lambda_{n}}\right) \ldots\left(j-1+\frac{1}{\lambda_{n}}\right)\left(\frac{x}{1+x}\right)^{j} \frac{1}{\lambda_{n}} j \lambda_{n}^{3} \\
& +(1+x)^{-\frac{1}{\lambda_{n}}} \sum_{j=0}^{\infty} \frac{1}{j !} \frac{1}{\lambda_{n}}\left(1+\frac{1}{\lambda_{n}}\right) \ldots\left(j-1+\frac{1}{\lambda_{n}}\right)\left(\frac{x}{1+x}\right)^{j} \frac{1}{\lambda_{n}} \frac{1}{3} \lambda_{n}^{3} \\
& =(1+x)^{-\frac{1}{\lambda_{n}}} \sum_{j=0}^{\infty} \frac{1}{j !} \frac{1}{\lambda_{n}}\left(1+\frac{1}{\lambda_{n}}\right) \ldots\left(j-1+\frac{1}{\lambda_{n}}\right)\left(\frac{x}{1+x}\right)^{j} j^{2} \lambda_{n}^{2} \\
& +(1+x)^{-\frac{1}{\lambda_{n}}} \sum_{j=0}^{\infty} \frac{1}{j !} \frac{1}{\lambda_{n}}\left(1+\frac{1}{\lambda_{n}}\right) \ldots\left(j-1+\frac{1}{\lambda_{n}}\right)\left(\frac{x}{1+x}\right)^{j} j \lambda_{n}^{2} \\
& +\frac{1}{3}(1+x)^{-\frac{1}{\lambda_{n}}} \sum_{j=0}^{\infty} \frac{1}{j !} \frac{1}{\lambda_{n}}\left(1+\frac{1}{\lambda_{n}}\right) \ldots\left(j-1+\frac{1}{\lambda_{n}}\right)\left(\frac{x}{1+x}\right)^{j} \lambda_{n}^{2} \\
& =V_{n}\left(e_{2} ; \lambda_{n}\right)(x)+\lambda_{n} V_{n}\left(e_{1} ; \lambda_{n}\right)(x)+\frac{1}{3} \lambda_{n}^{2} V_{n}\left(e_{0} ; \lambda_{n}\right)(x) \\
& =x^{2}+2 \lambda_{n} x+\lambda_{n} x^{2}+\frac{1}{3} \lambda_{n}^{2} .
\end{aligned}
$$

(ii) Finally, we get

$$
\begin{gathered}
K_{n}\left((t-x)^{2} ; \lambda_{n}\right)(x)=K_{n}\left(t^{2}-2 t x+x^{2} ; \lambda_{n}\right)(x) \\
=K_{n}\left(t^{2} ; \lambda_{n}\right)(x)-K_{n}\left(2 t x ; \lambda_{n}\right)(x)+K_{n}\left(x^{2} ; \lambda_{n}\right)(x) \\
=K_{n}\left(e_{2} ; \lambda_{n}\right)(x)-2 x K_{n}\left(e_{1} ; \lambda_{n}\right)(x)+x^{2} K_{n}\left(e_{0} ; \lambda_{n}\right)(x) \\
=x^{2}+2 \lambda_{n} x+\lambda_{n} x^{2}+\frac{1}{3} \lambda_{n}^{2}-2 x^{2}-x \lambda_{n}+x^{2}=\lambda_{n}\left(x+x^{2}+\frac{1}{3} \lambda_{n}\right) .
\end{gathered}
$$

The main result of this section is the following. 
Theorem 2.2. Let $\lambda_{n} \searrow 0$ (with $n \rightarrow \infty$ ) as fast we want and suppose that $f$ : $[0,+\infty) \rightarrow \mathbb{R}$ is uniformly continuous on $[0,+\infty)$. For all $x \in[0,+\infty)$ and $n \in \mathbb{N}$, we have

$$
\left|K_{n}\left(f ; \lambda_{n}\right)(x)-f(x)\right| \leq 2 \omega_{1}\left(f ; \sqrt{\lambda_{n}} \cdot \sqrt{x^{2}+x+\lambda_{n} / 3}\right),
$$

where $\omega_{1}(f ; \delta)=\sup \{|f(x)-f(y)| ; x, y \in \mathbb{R},|x-y| \leq \delta\}$ denotes the modulus of continuity of $f$ with the step $\delta$.

Proof. By the classical theory (see, e.g., Shisha-Mond [14] or, e.g., [1], Proposition 1.6.3) (where although the result is proved for continuous functions on compact intervals, the reasonings are similar for uniformly continuous functions on $[0,+\infty)$ ), for any positive and linear operator $L$ defined on the set of uniformly continuous functions $U C[0,+\infty)$, we obtain

$$
|L(f)(x)-f(x)| \leq\left(1+\delta^{-1} \sqrt{L\left(\varphi_{x}^{2}\right)(x)}\right) \omega(f ; \delta),
$$

for all $f \in U C[0,+\infty), x \in[0,+\infty), \delta>0$, where $\varphi_{x}(t)=|t-x|$.

Replacing above $L$ by $K_{n}$ and taking into account that by Lemma 2.1, (ii) we have

$$
\sqrt{K_{n}\left((t-x)^{2} ; \lambda_{n}\right)(x)}=\sqrt{\lambda_{n}\left(x+x^{2}+\frac{1}{3} \lambda_{n}\right)}=\sqrt{\lambda_{n}} \cdot \sqrt{x+x^{2}+\frac{1}{3} \lambda_{n}},
$$

this implies

$$
\left|K_{n}\left(f ; \lambda_{n}\right)(x)-f(x)\right| \leq\left(1+\delta^{-1} \sqrt{\lambda_{n}} \cdot \sqrt{x+x^{2}+\lambda_{n} / 3}\right) \omega_{1}(f ; \delta) .
$$

Choosing now here $\delta=\sqrt{\lambda_{n}} \cdot \sqrt{x^{2}+x+\lambda_{n} / 3}$ we get

$$
\left|K_{n}\left(f ; \lambda_{n}\right)(x)-f(x)\right| \leq 2 \omega_{1}\left(f ; \sqrt{\lambda_{n}} \sqrt{x+x^{2}+\lambda_{n} / 3}\right),
$$

which proves the estimate in the statement.

As an immediate consequence of Theorem 2.2 we get the following.

Corollary 2.3. Let $\lambda_{n} \searrow 0$ as fast we want and suppose that $f$ is a Lipschitz function, that is there exists $M>0$ such that $|f(x)-f(y)| \leq M|x-y|$, for all $x, y \in[0, \infty)$. Then, for all $x \in[0,+\infty)$ and $n \in \mathbb{N}$ we have

$$
\left|K_{n}\left(f ; \lambda_{n}\right)(x)-f(x)\right| \leq 2 M \sqrt{\lambda_{n}} \cdot \sqrt{x+x^{2}+\lambda_{n} / 3} .
$$

Proof. Since by hypothesis $f$ is a Lipschitz function, we easily get $\omega_{1}(f ; \delta) \leq M \delta$, for all $\delta>0$. Choosing now $\delta=\sqrt{\lambda_{n}} \cdot \sqrt{x+x^{2}+\lambda_{n} / 3}$ and applying Theorem 2.2, we get the desired estimate.

Remarks. 1) Since $f \in U C[0,+\infty)$, it is well-known that we get $\lim _{\delta \searrow_{0}} \omega_{1}(f ; \delta)=0$. Therefore, since $\lambda_{n} \searrow 0$, passing to limit with $n \rightarrow \infty$ in the estimate in Theorem 2.2, it follows that $K_{n}\left(f ; \lambda_{n}\right)(x) \rightarrow f(x)$, pointwise for any $x \in[0,+\infty)$. Now, in order to get uniform convergence in the above results, the expression $\sqrt{x+x^{2}+\lambda_{n} / 3}$ must be bounded, fact which holds when $x$ belongs to a compact subinterval of $[0,+\infty)$.

2) If $f \in U C[0,+\infty)$, then $K_{n}\left(f ; \lambda_{n}\right)(x)$ is well defined (that is $\left|K_{n}\left(f ; \lambda_{n}\right)(x)\right|<$ $+\infty$ for all $x \in[0,+\infty)$ and $n \in \mathbb{N})$. Indeed, if $f$ is uniformly continuous on $[0,+\infty)$ then it is well known that its growth on $[0,+\infty)$ is linear, i.e. there exist $\alpha, \beta>0$ such that $|f(x)| \leq \alpha x+\beta$, for all $x \in[0,+\infty$ ) (see e.g. [4], p. 48, Problème 4, or [5]). This immediately implies

$$
\left|K_{n}\left(f ; \lambda_{n}\right)(x)\right| \leq K_{n}\left(|f| ; \lambda_{n}\right)(x) \leq \alpha \cdot K_{n}\left(e_{1} ; \lambda_{n}\right)(x)+\beta=\alpha\left(x+\lambda_{n} / 2\right)+\beta,
$$


for all $x \in[0,+\infty), n \in \mathbb{N}$.

3) The optimality of the estimates in Theorem 2.2 and Corollary 2.3 consists in the fact that given an arbitrary sequence of strictly positive numbers $\left(\gamma_{n}\right)_{n}$, with $\lim _{n \rightarrow \infty} \gamma_{n}=0$, we always can find a sequence $\lambda_{n}$ satisfying

$$
2 \omega_{1}\left(f ; \sqrt{\lambda_{n}} \cdot \sqrt{x+x^{2}+\lambda_{n} / 3}\right) \leq \gamma_{n}
$$

for all $n \in \mathbb{N}$ and $x$ belonging to a compact subinterval of $[0,+\infty)$ in the case of Theorem 2.2 and $\left.\sqrt{\lambda_{n}} \cdot \sqrt{x+x^{2}+\lambda_{n} / 3}\right) \leq \gamma_{n}$ for all $n \in \mathbb{N}$ and $x$ in a compact subinterval of $[0,+\infty)$, in the case of Corollary 2.3.

\section{Generalized Szász-Kantorovich operators}

The formula for the classic, linear and positive Szász-Kantorovich operators is given by (see, e.g., [16])

$$
S_{n}(f)(x)=e^{-n x} \sum_{j=0}^{\infty} \frac{(n x)^{j}}{j !} n \int_{\frac{j}{n}}^{\frac{j+1}{n}} f(v) d v=e^{-n x} \sum_{j=0}^{\infty} \frac{(n x)^{j}}{j !} \int_{0}^{1} f\left(\frac{t+j}{n}\right) d t .
$$

Replacing above $n$ with $\frac{1}{\lambda_{n}}$, we obtain the generalized Szász-Kantorovich operators, defined by the formula

$$
\begin{aligned}
S_{n}\left(f ; \lambda_{n}\right)(x) & =e^{-\frac{x}{\lambda_{n}}} \sum_{j=0}^{\infty} \frac{x^{j}}{\lambda_{n}^{j} j !} \frac{1}{\lambda_{n}} \int_{j \lambda_{n}}^{(j+1) \lambda_{n}} f(v) d v \\
& =e^{-\frac{x}{\lambda_{n}}} \sum_{j=0}^{\infty} \frac{x^{j}}{\lambda_{n}^{j} j !} \int_{0}^{1} f\left(\lambda_{n}(t+j)\right) d t
\end{aligned}
$$

In this section we study the approximation properties of the operator $S_{n}\left(f ; \lambda_{n}\right)(x)$. Firstly we need the following lemma.

Lemma 3.1. We have:

(i) $S_{n}\left(e_{0} ; \lambda_{n}\right)(x)=1 ; S_{n}\left(e_{1} ; \lambda_{n}\right)(x)=x+\frac{1}{2} \cdot \lambda_{n}$;

$$
S_{n}\left(e_{2} ; \lambda_{n}\right)(x)=x^{2}+2 \lambda_{n} x+\frac{1}{3} \cdot \lambda_{n}^{2} ;
$$

(ii) $S_{n}\left((t-x)^{2} ; \lambda_{n}\right)(x)=\lambda_{n}\left(x+\frac{1}{3} \cdot \lambda_{n}\right)$.

Proof. (i) We have

$$
S_{n}\left(e_{0} ; \lambda_{n}\right)(x)=e^{-x / \lambda_{n}} \sum_{j=0}^{\infty} \frac{x^{j}}{j ! \lambda_{n}^{j}}=1,
$$

for all $x \geq 0$ and $n \in \mathbb{N}$. Then,

$$
\begin{aligned}
S_{n}\left(e_{1} ; \lambda_{n}\right)(x) & =e^{-x / \lambda_{n}} \sum_{j=0}^{\infty} \frac{x^{j}}{j ! \lambda_{n}^{j}} \frac{1}{\lambda_{n}} \cdot \frac{1}{2}\left\{\left[(j+1) \lambda_{n}\right]^{2}-\left(j \lambda_{n}\right)^{2}\right\} \\
& =e^{-x / \lambda_{n}} \sum_{j=0}^{\infty} \frac{x^{j}}{j ! \lambda_{n}^{j}} \frac{1}{2 \lambda_{n}} \cdot\left(2 \lambda_{n}^{2} j+\lambda_{n}^{2}\right)
\end{aligned}
$$




$$
\begin{gathered}
=e^{-x / \lambda_{n}} \sum_{j=0}^{\infty} \frac{x^{j}}{j ! \lambda_{n}^{j}} \frac{\lambda_{n}}{2}+\lambda_{n} \cdot e^{-x / \lambda_{n}} \sum_{j=0}^{\infty} \frac{x^{j} \cdot j}{j ! \lambda_{n}^{j}} \\
=\frac{\lambda_{n}}{2}+x \cdot e^{-x / \lambda_{n}} \sum_{j=1}^{\infty} \frac{x^{j-1}}{(j-1) ! \lambda_{n}^{j-1}}=\frac{\lambda_{n}}{2}+x \cdot e^{-x / \lambda_{n}} \sum_{k=0}^{\infty} \frac{x^{k}}{k ! \lambda_{n}^{k}}=\frac{\lambda_{n}}{2}+x .
\end{gathered}
$$

Also,

$$
\begin{gathered}
S_{n}\left(e_{2} ; \lambda_{n}\right)(x)=e^{-x / \lambda_{n}} \sum_{j=0}^{\infty} \frac{x^{j}}{j ! \lambda_{n}^{j}} \frac{1}{\lambda_{n}} \cdot \frac{1}{3}\left\{\left[(j+1) \lambda_{n}\right]^{3}-\left(j \lambda_{n}\right)^{3}\right\} \\
=e^{-x / \lambda_{n}} \sum_{j=0}^{\infty} \frac{x^{j}}{j ! \lambda_{n}^{j}} \frac{1}{3 \lambda_{n}} \cdot\left\{\left(3 j^{2}+3 j+1\right) \lambda_{n}^{3}\right\} \\
=\frac{\lambda_{n}^{2}}{3}+e^{-x / \lambda_{n}} \sum_{j=1}^{\infty} \frac{x^{j} j^{2} \lambda_{n}^{2}}{j ! \lambda_{n}^{j}}+e^{-x / \lambda_{n}} \sum_{j=1}^{\infty} \frac{x^{j} j \lambda_{n}^{2}}{j ! \lambda_{n}^{j}} \\
=\frac{\lambda_{n}^{2}}{3}+e^{-x / \lambda_{n}} \sum_{j=1}^{\infty} \frac{x^{j} j(j-1) \lambda_{n}^{2}}{j ! \lambda_{n}^{j}}+2 e^{-x / \lambda_{n}} \sum_{j=1}^{\infty} \frac{x^{j} j \lambda_{n}^{2}}{j ! \lambda_{n}^{j}}=x^{2}+2 x \lambda_{n}+\frac{\lambda_{n}^{2}}{3} .
\end{gathered}
$$

(ii) Concluding, we get

$$
\begin{gathered}
S_{n}\left((\cdot-x)^{2} ; \lambda_{n}\right)(x)=K_{n}\left(\lambda_{n} ; e_{2}\right)(x)-2 x \cdot K_{n}\left(\lambda_{n} ; e_{1}\right)(x)+x^{2} \\
=x \lambda_{n}+\lambda_{n}^{2} / 3=\lambda_{n}\left(x+\lambda_{n} / 3\right) .
\end{gathered}
$$

The main result of this section is the following.

Theorem 3.2. Let $\lambda_{n} \searrow 0$ (with $n \rightarrow \infty$ ) as fast we want and suppose that $f$ : $[0,+\infty) \rightarrow \mathbb{R}$ is uniformly continuous on $[0,+\infty)$. For all $x \in[0,+\infty)$ and $n \in \mathbb{N}$, we have

$$
\left|S_{n}\left(f ; \lambda_{n}\right)(x)-f(x)\right| \leq 2 \omega_{1}\left(f ; \sqrt{\lambda_{n}} \cdot \sqrt{x+\lambda_{n} / 3}\right),
$$

where $\omega_{1}(f ; \delta)=\sup \{|f(x)-f(y)| ; x, y \in \mathbb{R},|x-y| \leq \delta\}$ denotes the modulus of continuity of $f$ with the step $\delta$.

Proof. Reasoning exactly as in the proof of Theorem 2.2, we can write

$$
\left|S_{n}\left(f ; \lambda_{n}\right)(x)-f(x)\right| \leq\left(1+\delta^{-1} \sqrt{S_{n}\left(\varphi_{x}^{2} ; \lambda_{n}\right)(x)}\right) \omega_{1}(f ; \delta) .
$$

Choosing here $\delta=\sqrt{S_{n}\left(\varphi_{x}^{2} ; \lambda_{n}\right)(x)}$ an using Lemma 3.1, (ii), we obtain

$$
\left|S_{n}\left(f ; \lambda_{n}\right)(x)-f(x)\right| \leq 2 \omega_{1}\left(f ; \sqrt{\lambda_{n}} \cdot \sqrt{x+\frac{1}{3} \lambda_{n}}\right) \leq 2 \omega_{1}\left(f ; \sqrt{\lambda_{n}} \cdot \sqrt{x+\frac{1}{3} \lambda_{n}}\right),
$$

which proves the theorem.

As an immediate consequence of Theorem 3.2 we get the following.

Corollary 3.3. Let $\lambda_{n} \searrow 0$ as fast we want and suppose that $f$ is a Lipschitz function, that is there exists $M>0$ such that $|f(x)-f(y)| \leq M|x-y|$, for all $x, y \in[0, \infty)$. Then, for all $x \in[0,+\infty)$ and $n \in \mathbb{N}$ we have

$$
\left|S_{n}\left(f ; \lambda_{n}\right)(x)-f(x)\right| \leq 2 M \sqrt{\lambda_{n}} \cdot \sqrt{x+\lambda_{n} / 3} .
$$


Proof. Since by hypothesis $f$ is a Lipschitz function, we easily get $\omega_{1}(f ; \delta) \leq M \delta$, for all $\delta>0$. Choosing now $\delta=\sqrt{\lambda_{n}} \cdot \sqrt{x+\lambda_{n} / 3}$ and applying Theorem 3.2, we get the desired estimate.

Remark. All the Remarks 1)-3) made at the end of the previous section remain valid for the generalized Szász-Kantorovich operators too.

\section{Generalized Szász-Durrmeyer-type operators}

Let us recall that the classical Szász-Durrmeyer operators are given by the formula (see, e.g., [13])

$$
S D_{n}(f)(x)=n \sum_{j=0}^{\infty} s_{n, j}(x) \int_{0}^{\infty} s_{n, j}(t) f(t) d t,
$$

where $s_{n, j}(x)=e^{-n x} \frac{(n x)^{j}}{j !}$.

If we replace $n$ with $\frac{1}{\lambda_{n}}$, then we obtain the generalized Szász-Durrmeyer operators, defined by the formula

$$
S D_{n}\left(f ; \lambda_{n}\right)(x)=\frac{1}{\lambda_{n}} \sum_{j=0}^{\infty} e^{-\frac{x}{\lambda_{n}}} \cdot \frac{x^{j}}{\lambda_{n}^{j} j !} \int_{0}^{\infty} e^{-\frac{t}{\lambda_{n}}} \cdot \frac{t^{j}}{\lambda_{n}^{j} j !} f(t) d t .
$$

In the first part of this section we study the approximation properties of the operator $S D_{n}\left(f ; \lambda_{n}\right)(x)$. Firstly we need the following lemma.

Lemma 4.1. We have:

(i) $S D_{n}\left(e_{0} ; \lambda_{n}\right)(x)=1 ; S D_{n}\left(e_{1} ; \lambda_{n}\right)(x)=x+\lambda_{n}$;

$$
S D_{n}\left(e_{2} ; \lambda_{n}\right)(x)=x^{2}+4 \lambda_{n} x+2 \lambda_{n}^{2}
$$

(ii) $S D_{n}\left((t-x)^{2} ; \lambda_{n}\right)(x)=\lambda_{n}\left(2 x+2 \lambda_{n}\right)$.

Proof. (i) Denoting

we can write

$$
I_{j}(f)=\int_{0}^{\infty} e^{-\frac{t}{\lambda_{n}}} \frac{\left(\frac{t}{\lambda_{n}}\right)^{j}}{j !} f(t) d t
$$

$$
S D_{n}\left(f ; \lambda_{n}\right)(x)=\frac{1}{\lambda_{n}} \sum_{j=0}^{\infty} e^{-\frac{x}{\lambda_{n}}} \frac{\left(\frac{x}{\lambda_{n}}\right)^{j}}{j !} \cdot I_{j}(f) .
$$

Now, taking $f(t)=t^{p}$ and making the change of variable $v=\frac{t}{\lambda_{n}}$ it follows

$$
\begin{aligned}
I_{j}\left(e_{p}\right)= & \lambda_{n} \int_{0}^{\infty} e^{-v} \cdot \frac{v^{j}}{j !} \cdot \lambda_{n}^{p} \cdot v^{p} d v=\frac{\lambda_{n}^{p+1}}{j !} \cdot \int_{0}^{\infty} e^{-v} v^{p+j} d v \\
& =\frac{\lambda_{n}^{p+1}}{j !} \cdot \Gamma(p+j+1-1)=\frac{\lambda_{n}^{p+1}}{j !} \cdot(p+j) !,
\end{aligned}
$$

where $\Gamma$ is the Euler's gamma function.

So, for $p=0$, we have $I_{j}\left(e_{0}\right)=\frac{\lambda_{n}}{j !} j !=\lambda_{n}$, which implies

$$
S D_{n}\left(e_{0}, \lambda_{n}\right)(x)=\frac{1}{\lambda_{n}} \sum_{j=0}^{\infty} e^{-\frac{x}{\lambda_{n}}} \frac{\left(\frac{x}{\lambda_{n}}\right)^{j}}{j !} \lambda_{n}=\frac{1}{\lambda_{n}} \lambda_{n}=1 .
$$


Now, for $p=1$ we have $I_{j}\left(e_{1}\right)=\frac{\left(\lambda_{n}\right)^{2}}{j !}(j+1) !=(j+1) \lambda_{n}^{2}$, which implies

$$
\begin{aligned}
& S D_{n}\left(e_{1}, \lambda_{n}\right)(x)=\frac{1}{\lambda_{n}} \sum_{j=0}^{\infty} e^{-\frac{x}{\lambda_{n}}} \frac{\left(\frac{x}{\lambda_{n}}\right)^{j}}{j !}(j+1) \lambda_{n}^{2} \\
& =\frac{1}{\lambda_{n}} \sum_{j=0}^{\infty} e^{-\frac{x}{\lambda_{n}}} \frac{\left(\frac{x}{\lambda_{n}}\right)^{j}}{j !} j \lambda_{n}^{2}+\frac{1}{\lambda_{n}} \sum_{j=0}^{\infty} e^{-\frac{x}{\lambda_{n}}} \frac{\left(\frac{x}{\lambda_{n}}\right)^{j}}{j !} \lambda_{n}^{2}=x+\lambda_{n} .
\end{aligned}
$$

Finally, for $p=2$, we have $I_{j}\left(e_{2}\right)=\frac{\left(\lambda_{n}\right)^{3}}{j !}(j+2) !=(j+1)(j+2) \lambda_{n}^{3}$, which implies

$$
\begin{aligned}
& S D_{n}\left(e_{2}, \lambda_{n}\right)(x)=\frac{1}{\lambda_{n}} \sum_{j=0}^{\infty} e^{-\frac{x}{\lambda_{n}}} \frac{\left(\frac{x}{\lambda_{n}}\right)^{j}}{j !}\left(j^{2}+3 j+2\right) \lambda_{n}^{3} \\
& =\frac{1}{\lambda_{n}} \sum_{j=0}^{\infty} e^{-\frac{x}{\lambda_{n}}} \frac{\left(\frac{x}{\lambda_{n}}\right)^{j}}{j !} j^{2} \lambda_{n}^{3}+\frac{1}{\lambda_{n}} \sum_{j=0}^{\infty} e^{-\frac{x}{\lambda_{n}}} \frac{\left(\frac{x}{\lambda_{n}}\right)^{j}}{j !} 3 j \lambda_{n}^{3} \\
& +\frac{1}{\lambda_{n}} \sum_{j=0}^{\infty} e^{-\frac{x}{\lambda_{n}}} \frac{\left(\frac{x}{\lambda_{n}}\right)^{j}}{j !} 2 \lambda_{n}^{3}=\left(x^{2}+\lambda_{n} x\right)+3 \lambda_{n} x+2 \lambda_{n}^{2}=x^{2}+4 \lambda_{n} x+2 \lambda_{n}^{2} .
\end{aligned}
$$

(ii) Concluding, we get

$$
\begin{gathered}
S D_{n}\left((t-x)^{2} ; \lambda_{n}\right)(x)=S D_{n}\left(t^{2}, \lambda_{n}\right)(x)-S D_{n}\left(2 t x, \lambda_{n}\right)(x)+S D_{n}\left(x^{2}, \lambda_{n}\right)(x) \\
=x^{2}+4 \lambda_{n} x+2 \lambda_{n}^{2}-2 x\left(x+\lambda_{n}\right)+x^{2}=2 \lambda_{n} x+2 \lambda_{n}^{2},
\end{gathered}
$$

which proves the lemma.

The first main result of this section is the following.

Theorem 4.2. Let $\lambda_{n} \searrow 0$ as fast we want and suppose that $f:[0,+\infty) \rightarrow \mathbb{R}$ is uniformly continuous on $[0,+\infty)$. For all $x \in[0,+\infty)$ and $n \in \mathbb{N}$, we have

$$
\left|S D_{n}\left(f ; \lambda_{n}\right)(x)-f(x)\right| \leq 2 \omega_{1}\left(f ; \sqrt{\lambda_{n}} \cdot \sqrt{2 x+2 \lambda_{n}}\right) .
$$

Proof. Reasoning exactly as in the proof of Theorem 2.2, we can write

$$
\left|S D_{n}\left(f ; \lambda_{n}\right)(x)-f(x)\right| \leq\left(1+\delta^{-1} \sqrt{S D_{n}\left(\varphi_{x}^{2} ; \lambda_{n}\right)(x)}\right) \omega_{1}(f ; \delta) .
$$

Choosing here $\delta=\sqrt{S D_{n}\left(\varphi_{x}^{2} ; \lambda_{n}\right)(x)}$ and using Lemma 4.1, (ii), we obtain

$$
\left|S_{n}\left(f ; \lambda_{n}\right)(x)-f(x)\right| \leq 2 \omega_{1}\left(f ; \sqrt{\lambda_{n}} \cdot \sqrt{2 x+2 \lambda_{n}}\right),
$$

which proves the theorem.

As an immediate consequence of Theorem 4.2 we get the following.

Corollary 4.3. Let $\lambda_{n} \searrow 0$ as fast we want and suppose that $f$ is a Lipschitz function, that is there exists $M>0$ such that $|f(x)-f(y)| \leq M|x-y|$, for all $x, y \in[0, \infty)$. Then, for all $x \in[0,+\infty)$ and $n \in \mathbb{N}$ we have

$$
\left|S_{n}\left(f ; \lambda_{n}\right)(x)-f(x)\right| \leq 2 M \sqrt{\lambda_{n}} \cdot \sqrt{2 x+2 \lambda_{n}} .
$$

Proof. Since by hypothesis $f$ is a Lipschitz function, we easily get $\omega_{1}(f ; \delta) \leq M \delta$, for all $\delta>0$. Choosing now $\delta=\sqrt{\lambda_{n}} \cdot \sqrt{2 x+2 \lambda_{n}}$ and applying Theorem 4.2, we get the desired estimate.

Remark. All the Remarks 1)-3) made at the end of Section 2 remain valid for the generalized Szász-Durrmeyer, $S D_{n}\left(f ; \lambda_{n}\right)$, operators too. 
In what follows we will introduce and study the generalized Szász-DurrmeyerStancu operators. Thus it is well-known that the classical Szász-Durrmeyer-Stancu operators are given by the formula (see, e.g., [8])

$$
S D_{n}^{(\alpha, \beta)}(f)(x)=n \sum_{j=0}^{\infty} s_{n, j}(x) \int_{0}^{\infty} s_{n, j}(t) f\left(\frac{n t+\alpha}{n+\beta}\right) d t
$$

where $0 \leq \alpha \leq \beta$ and $s_{n, j}(x)=e^{-n x} \frac{(n x)^{j}}{j !}$.

If we replace $n$ with $\frac{1}{\lambda_{n}}$, we obtain:

$$
S D_{n}^{(\alpha, \beta)}\left(f ; \lambda_{n}\right)(x)=\frac{1}{\lambda_{n}} \sum_{j=0}^{\infty} e^{-\frac{x}{\lambda_{n}}} \frac{\left(\frac{x}{\lambda_{n}}\right)^{j}}{j !} \int_{0}^{\infty} e^{-\frac{x}{\lambda_{n}}} \frac{\left(\frac{x}{\lambda_{n}}\right)^{j}}{j !} f\left(\frac{\frac{t}{\lambda_{n}}+\alpha}{\frac{1}{\lambda_{n}+\beta}}\right) d t
$$

Firstly we prove the following lemma.

Lemma 4.4. We have:

(i) $S D_{n}^{(\alpha, \beta)}\left(e_{0} ; \lambda_{n}\right)(x)=1 ; S D_{n}^{(\alpha, \beta)}\left(e_{1} ; \lambda_{n}\right)(x)=\frac{x}{1+\lambda_{n} \beta}+\frac{\lambda_{n}(\alpha+1)}{1+\lambda_{n} \beta}$;

$$
S D_{n}^{(\alpha, \beta)}\left(e_{2} ; \lambda_{n}\right)(x)=\frac{x^{2}}{\left(1+\lambda_{n} \beta\right)^{2}}+\frac{\lambda_{n}(2 \alpha+3)}{\left(1+\lambda_{n} \beta\right)^{2}} x+\frac{\lambda_{n}^{2}\left(\alpha^{2}+2 \alpha+2\right)}{\left(1+\lambda_{n} \beta\right)^{2}}
$$

(ii) $S D_{n}^{(\alpha, \beta)}\left((t-x)^{2} ; \lambda_{n}\right)(x)$

$$
=\frac{\lambda_{n}^{2} \beta^{2}}{\left(1+\lambda_{N} \beta\right)^{2}} x^{2}+\frac{\lambda_{n}\left(1-2 \beta(\alpha+1) \lambda_{n}\right)}{\left(1+\lambda_{n} \beta\right)^{2}} x+\frac{\lambda_{n}^{2}\left(\alpha^{2}+2 \alpha+2\right)}{\left(1+\lambda_{n} \beta\right)^{2}} .
$$

Proof. (i) Firstly, we calculate $T_{n, k}^{(\alpha, \beta)}(x):=S D_{n}^{(\alpha, \beta)}\left(e_{k}\right)(x), k=0,1,2$. For this purpose, we will use the following formula in Lemma 2.1 in [8]

$$
T_{n, k}^{(\alpha, \beta)}=\sum_{j=0}^{k}\left(\begin{array}{l}
k \\
j
\end{array}\right) \frac{n^{j} \alpha^{k-j}}{(n+\beta)^{k}} T_{n, j}(x),
$$

where $T_{n, k}(x)=S D_{n}\left(e_{k}\right)(x)$.

Therefore, before that we need to calculate $T_{n, k}(x)$. For the calculation of $T_{n, k}(x)$, we use the recurrence formula in Lemma 2.2 in [7]

$$
T_{n, k}^{\prime}(x)=\frac{n}{x} T_{n, k+1}(x)-\left(n+\frac{k+1}{x}\right) T_{n, k}(x),
$$

taking into account that $T_{n, 0}(x)=1$.

Thus, taking in (4.2) $k=0$ we immediately get

$$
0=\frac{n}{x} T_{n, 1}(x)-(n+1 / x) T_{n, 0}(x),
$$

which implies

$$
T_{n, 1}(x)=(n+1 / x) \cdot \frac{x}{n}=x+1 / n .
$$

Taking in (4.2) $k=1$, it follows

$$
1=\frac{n}{x} T_{n, 2}(x)-\left(n+\frac{2}{x}\right)\left(x+\frac{1}{n}\right),
$$


which implies

$$
T_{n, 2}(x)=\left(n x+3+\frac{2}{n x}\right)\left(x+\frac{1}{n}\right)=x^{2}+\frac{3 x}{n}+\frac{2}{n^{2}} .
$$

Returning now to the formula (4.1), for $k=0$ we obtain $T_{n, 0}^{(\alpha, \beta)}(x)=1$, for $k=1$ we obtain

$$
\begin{gathered}
T_{n, 1}^{(\alpha, \beta)}=\sum_{j=0}^{1}\left(\begin{array}{l}
1 \\
j
\end{array}\right) \frac{n^{j} \alpha^{1-j}}{(n+\beta)^{1}} T_{n, j}(x)=\frac{\alpha}{n+\beta}+\frac{n}{n+\beta}\left(x+\frac{1}{n}\right) \\
=\frac{n}{n+\beta} x+\frac{\alpha+1}{n+\beta},
\end{gathered}
$$

while for $k=1$ we get

$$
\begin{gathered}
T_{n, 2}^{(\alpha, \beta)}(x)=\sum_{j=0}^{2}\left(\begin{array}{l}
2 \\
j
\end{array}\right) \frac{n^{j} \alpha^{2-j}}{(n+\beta)^{2}} T_{n, j}(x) \\
=\frac{\alpha^{2}}{(n+\beta)^{2}}+\frac{2 n \alpha}{(n+\beta)^{2}}\left(x+\frac{1}{n}\right)+\frac{n^{2}}{(n+\beta)^{2}}\left(x^{2}+\frac{3 x}{n}+\frac{2}{n^{2}}\right) \\
=\frac{n^{2}}{(n+\beta)^{2}} x^{2}+\frac{n(2 \alpha+3)}{(n+\beta)^{2}} x+\frac{\alpha^{2}+2 \alpha+2}{(n+\beta)^{2}} .
\end{gathered}
$$

Now, if we replace $n$ with $\frac{1}{\lambda_{n}}$ we easily obtain

$$
\begin{gathered}
S D_{n}^{(\alpha, \beta)}\left(e_{0} ; \lambda_{n}\right)(x)=1, \\
S D_{n}^{(\alpha, \beta)}\left(e_{1} ; \lambda_{n}\right)(x)=\frac{x}{1+\lambda_{n} \beta}+\frac{\lambda_{n}(\alpha+1)}{1+\lambda_{n} \beta}, \\
S D_{n}^{(\alpha, \beta)}\left(e_{2} ; \lambda_{n}\right)(x)=\frac{x^{2}}{\left(1+\lambda_{n} \beta\right)^{2}}+\frac{\lambda_{n}(2 \alpha+3)}{\left(1+\lambda_{n} \beta\right)^{2}} x+\frac{\lambda_{n}^{2}\left(\alpha^{2}+2 \alpha+2\right)}{\left(1+\lambda_{n} \beta\right)^{2}} .
\end{gathered}
$$

(ii) We have

$$
\begin{gathered}
S D_{n}^{(\alpha, \beta)}\left((t-x)^{2} ; \lambda_{n}\right)(x) \\
=S D_{n}^{(\alpha, \beta)}\left(e_{2} ; \lambda_{n}\right)(x)-2 x S D_{n}^{(\alpha, \beta)}\left(e_{1} ; \lambda_{n}\right)(x)+x^{2} S D_{n}^{(\alpha, \beta)}\left(e_{0} ; \lambda_{n}\right)(x) \\
=x^{2}\left[\frac{1}{\left(1+\lambda_{n} \beta\right)^{2}}-\frac{2}{1+\lambda_{n} \beta}+1\right]+x\left[\frac{\lambda_{n}(2 \alpha+3)}{\left(1+\lambda_{n} \beta\right)^{2}}-\frac{2 \lambda_{n}(\alpha+1)}{1+\lambda_{n} \beta}\right] \\
+\frac{\lambda_{n}^{2}\left(\alpha^{2}+2 \alpha+2\right)}{\left(1+\lambda_{n} \beta\right)^{2}}=\frac{\lambda_{n}^{2} \beta^{2}}{\left(1+\lambda_{n} \beta\right)^{2}} x^{2}+\frac{\lambda_{n}\left(1-2 \beta(\alpha+1) \lambda_{n}\right)}{\left(1+\lambda_{n} \beta\right)^{2}} x \\
+\frac{\lambda_{n}^{2}\left(\alpha^{2}+2 \alpha+2\right)}{\left(1+\lambda_{n} \beta\right)^{2}}
\end{gathered}
$$

which proves the lemma.

The second main result of this section is the following.

Theorem 4.5. Let $0 \leq \alpha \leq \beta, \lambda_{n} \searrow 0$ as fast we want and suppose that $f:[0,+\infty) \rightarrow$ $\mathbb{R}$ is uniformly continuous on $[0,+\infty)$. For all $x \in[0,+\infty)$ and $n \in \mathbb{N}$, we have

$$
\left|S D_{n}^{(\alpha, \beta)}\left(f ; \lambda_{n}\right)(x)-f(x)\right| \leq 2 \omega_{1}\left(f ; \sqrt{\lambda_{n}} \cdot \sqrt{E_{n}^{(\alpha, \beta)}(x)}\right),
$$


where

$$
E_{n}^{(\alpha, \beta)}(x)=\frac{\lambda_{n} \beta^{2}}{\left(1+\lambda_{n} \beta\right)^{2}} x^{2}+\frac{1-2 \beta(\alpha+1) \lambda_{n}}{\left(1+\lambda_{n} \beta\right)^{2}} x+\frac{\lambda_{n}\left(\alpha^{2}+2 \alpha+2\right)}{\left(1+\lambda_{n} \beta\right)^{2}} .
$$

Proof. Reasoning exactly as in the proof of Theorem 2.2, we can write

$$
\left|S D_{n}^{(\alpha, \beta)}\left(f ; \lambda_{n}\right)(x)-f(x)\right| \leq\left(1+\delta^{-1} \sqrt{S D_{n}^{(\alpha, \beta)}\left(\varphi_{x}^{2} ; \lambda_{n}\right)(x)}\right) \omega_{1}(f ; \delta) .
$$

Choosing here $\delta=\sqrt{S D_{n}^{(\alpha, \beta)}\left(\varphi_{x}^{2} ; \lambda_{n}\right)(x)}$ and using Lemma 4.1, (ii), we obtain

$$
\left|S D_{n}^{(\alpha, \beta)}\left(f ; \lambda_{n}\right)(x)-f(x)\right| \leq 2 \omega_{1}\left(f ; \sqrt{\lambda_{n}} \cdot \sqrt{E_{n}^{(\alpha, \beta)}(x)}\right),
$$

which proves the theorem.

As an immediate consequence of Theorem 4.5 we get the following.

Corollary 4.6. Let $0 \leq \alpha \leq \beta, \lambda_{n} \searrow 0$ as fast we want and suppose that $f$ is a Lipschitz function, that is there exists $M>0$ such that $|f(x)-f(y)| \leq M|x-y|$, for all $x, y \in[0, \infty)$. Then, for all $x \in[0,+\infty)$ and $n \in \mathbb{N}$ we have

$$
\left|S D_{n}^{(\alpha, \beta)}\left(f ; \lambda_{n}\right)(x)-f(x)\right| \leq 2 M \sqrt{\lambda_{n}} \cdot \sqrt{E_{n}^{(\alpha, \beta)}(x)} .
$$

Proof. Since by hypothesis $f$ is a Lipschitz function, we easily get $\omega_{1}(f ; \delta) \leq M \delta$, for all $\delta>0$. Choosing now $\delta=\sqrt{\lambda_{n}} \cdot \sqrt{E_{n}^{(\alpha, \beta)}(x)}$ and applying Theorem 4.5 , we get the desired estimate.

Remark. All the Remarks 1)-3) made at the end of Section 2 remain valid for the generalized Szász-Durrmeyer-Stancu, $S D_{n}^{(\alpha, \beta)}\left(f ; \lambda_{n}\right)$, operators too.

\section{Generalized Baskakov-Szász-Durrmeyer-Stancu operators}

For $0 \leq \alpha \leq \beta$, the classical Baskakov- Szász-Durrmeyer-Stancu operators are given by the formula (see, e.g., [12])

$$
V_{n}^{(\alpha, \beta)}(f)(x)=n \sum_{j=0}^{\infty} b_{n, j}(x) \int_{0}^{\infty} s_{n, j}(t) f\left(\frac{n t+\alpha}{n+\beta} d t,\right.
$$

where, $s_{n, j}(x)=e^{-n x} \frac{(n x)^{j}}{j !}$ and

$$
b_{n, j}(x)=\left(\begin{array}{c}
n+j-1 \\
j
\end{array}\right) \frac{x^{j}}{(1+x)^{n+j}}=(1+x)^{-n} \frac{n(n+1) \ldots(n+j-1)}{j !} \frac{x^{j}}{(1+x)^{j}} .
$$

If we replace $n$ with $\frac{1}{\lambda_{n}}$ we obtain the formula:

$$
\begin{aligned}
V_{n}^{(\alpha, \beta)}\left(f ; \lambda_{n}\right)(x)= & \frac{1}{\lambda_{n}} \sum_{j=0}^{\infty}(1+x)^{-\frac{1}{\lambda_{n}}} \frac{\frac{1}{\lambda_{n}}\left(\frac{1}{\lambda_{n}}+1\right) \ldots\left(\frac{1}{\lambda_{n}}+j-1\right)}{j !} \frac{x^{j}}{(1+x)^{j}} \\
& \cdot \int_{0}^{\infty} e^{-\frac{t}{\lambda_{n}}} \cdot \frac{\left(\frac{t}{\lambda_{n}}\right)^{j}}{j !} f\left(\frac{\frac{t}{\lambda_{n}}+\alpha}{\frac{1}{\lambda_{n}}+\beta}\right) d t .
\end{aligned}
$$

Firstly we need the following auxiliary result. 
Lemma 5.1. We have:

$$
\begin{aligned}
& \text { (i) } V_{n}^{(\alpha, \beta)}\left(e_{0} ; \lambda_{n}\right)(x)=1 ; V_{n}^{(\alpha, \beta)}\left(e_{1} ; \lambda_{n}\right)(x)=\frac{1}{1+\lambda_{n} \beta} x+\frac{\lambda_{n}+\lambda_{n} \alpha}{1+\lambda_{n} \beta} ; \\
& V_{n}^{(\alpha, \beta)}\left(e_{2} ; \lambda_{n}\right)(x)=\frac{1+\lambda_{n}}{\left(1+\lambda_{n} \beta\right)^{2}} x^{2}+\frac{4 \lambda_{n}+2 \lambda_{n} \alpha}{\left(1+\lambda_{n} \beta\right)^{2}} x+\frac{\lambda_{n}^{2} \alpha^{2}+2 \lambda_{n}^{2} \alpha+2 \lambda_{n}^{2}}{\left(1+\lambda_{n} \beta\right)^{2}} ; \\
& \text { (ii) } V_{n}^{(\alpha, \beta)}\left((t-x)^{2} ; \lambda_{n}\right)(x) \\
& \quad=\frac{\lambda_{n}+\lambda_{n}^{2} \beta^{2}}{\left(1+\lambda_{n} \beta\right)^{2}} x^{2}+\frac{2 \lambda_{n}-2 \lambda_{n}^{2} \beta-2 \lambda_{n}^{2} \alpha \beta}{\left(1+\lambda_{n} \beta\right)^{2}} x+\frac{\lambda_{n}^{2} \alpha^{2}+2 \lambda_{n}^{2} \alpha+2 \lambda_{n}^{2}}{\left(1+\lambda_{n} \beta\right)^{2}} .
\end{aligned}
$$

Proof. (i) We will make the calculations in three steps:

Step 1. We calculate $U_{n, k}^{(0,0)}(x):=V_{n}^{(0,0)}\left(e_{k}\right)(x), k=1,2$ by using the recurrence formula (see, e.g., Lemma 2 in [11])

$$
U_{n, k}^{(0,0)}(x)=\frac{x(1+x)}{n} \cdot\left[U_{n, k}^{(0,0)}(x)\right]^{\prime}+\frac{n x+k+1}{n} U_{n, k}^{(0,0)}(x),
$$

and by taking into account that $U_{n, 0}^{(0,0)}(x)=1$. Taking $k=0$ in (5.1), we obtain

$$
U_{n, 1}^{(0,0)}(x)=\frac{x(1+x)}{n} \cdot(1)^{\prime}+\frac{n x+1}{n} \cdot 1=\frac{n x+1}{n}=x+\frac{1}{n} .
$$

For $k=1$ in $(5.1)$, we get

$$
\begin{gathered}
U_{n, 2}^{(0,0)}(x)=\frac{x(1+x)}{n} \cdot\left(\frac{n x+1}{n}\right)^{\prime}+\frac{n x+2}{n} \cdot \frac{n x+1}{n}=\frac{x(1+x)}{n}+\frac{(n x+2)(n x+1)}{n^{2}} \\
=\frac{n x(1+x)+(n x+1)(n x+2)}{n^{2}}=x^{2}+\frac{x^{2}+4 x}{n}+\frac{2}{n^{2}} .
\end{gathered}
$$

Step 2. By direct calculation and based on the results obtained at Step 1, we will obtain the values for $V_{n}^{(\alpha, \beta)}\left(e_{k}\right)(x):=U_{n, k}^{(\alpha, \beta)}(x), k=0,1,2$. Indeed, based on the formulas

$$
\frac{n t+\alpha}{n+\beta}=\frac{n}{n+\beta} t+\frac{\alpha}{n+\beta}, U_{n, k}^{(\alpha, \beta)}(x)=n \sum_{j=0}^{\infty} b_{n, j}(x) \int_{0}^{\infty} s_{n, j}(t) f\left(\frac{n t+\alpha}{n+\beta}\right) d t
$$

for $k=0$ in $(5.2)$ we obtain

$$
U_{n, 0}^{(\alpha, \beta)}(x)=V_{n}^{(\alpha, \beta)}\left(e_{0}\right)(x)=n \sum_{j=0}^{\infty} b_{n, j}(x) \int_{0}^{\infty} s_{n, j}(t) d t=U_{n, 0}^{(0,0)}(x)=1 .
$$

Then, for $k=1$ in (5.2) it follows

$$
\begin{gathered}
U_{n, 1}^{(\alpha, \beta)}(x)=n \sum_{j=0}^{\infty} b_{n, j}(x) \int_{0}^{\infty} s_{n, j}(t) \frac{n t+\alpha}{n+\beta} d t \\
=n \sum_{j=0}^{\infty} b_{n, j}(x) \int_{0}^{\infty} s_{n, j}(t) \frac{n}{n+\beta} t d t+\frac{\alpha}{n+\beta} n \sum_{j=0}^{\infty} b_{n, j}(x) \int_{0}^{\infty} s_{n, j}(t) d t \\
=\frac{n}{n+\beta}\left(n \sum_{j=0}^{\infty} b_{n, j}(x) \int_{0}^{\infty} s_{n, j}(t) t d t\right)+\frac{\alpha}{n+\beta} \cdot U_{n, 0}^{(0,0)}(x)
\end{gathered}
$$




$$
=\frac{n}{n+\beta} \cdot U_{n, 1}^{(0,0)}(x)+\frac{\alpha}{n+\beta}=\frac{n}{n+\beta} \frac{n x+1}{n}+\frac{\alpha}{n+\beta}=\frac{n}{n+\beta} x+\frac{\alpha+1}{n+\beta} .
$$

Finally, for $k=2$ in (5.2) we obtain

$$
\begin{gathered}
U_{n, 2}^{(\alpha, \beta)}(x)=V_{n}^{(\alpha, \beta)}\left(e_{2}\right)(x)=n \sum_{j=0}^{\infty} b_{n, j}(x) \int_{0}^{\infty} s_{n, j}(t)\left(\frac{n t+\alpha}{n+\beta}\right)^{2} d t \\
=\frac{n^{2}}{(n+\beta)^{2}}\left(n \sum_{j=0}^{\infty} b_{n, j}(x) \int_{0}^{\infty} s_{n, j}(t) t^{2} d t\right) \\
+\frac{2 n \alpha}{(n+\beta)^{2}}\left(n \sum_{j=0}^{\infty} b_{n, j}(x) \int_{0}^{\infty} s_{n, j}(t) t d t\right) \\
+\frac{\alpha^{2}}{(n+\beta)^{2}}\left(n \sum_{j=0}^{\infty} b_{n, j}(x) \int_{0}^{\infty} s_{n, j}(t) d t\right) \\
=\frac{n^{2}}{(n+\beta)^{2}} \cdot U_{n, 2}^{(0,0)}(x)+\frac{2 n \alpha}{(n+\beta)^{2}} \cdot U_{n, 1}^{(0,0)}(x)+\frac{\alpha^{2}}{(n+\beta)^{2}} \cdot U_{n, 0}^{(0,0)}(x) \\
\frac{n x(1+x)+(n x+1)(n x+2)}{n^{2}}+\frac{2 n \alpha}{(n+\beta)^{2}} \frac{n x+1}{n}+\frac{n)^{2}}{(n+\beta)^{2}} \\
=\frac{n x(1+x)+(n x+1)(n x+2)+2 \alpha(n x+1)+\alpha^{2}}{(n+\beta)^{2}} \\
=\frac{n^{2}+n}{(n+\beta)^{2}} x^{2}+\frac{4 n+2 \alpha n}{(n+\beta)^{2}} x+\frac{\alpha^{2}+2 \alpha+2}{(n+\beta)^{2}} .
\end{gathered}
$$

Step 3. We calculate $U_{n, k}^{(\alpha, \beta)}(x), k=0,1,2$, by replacing at Step $2, n$ with $\frac{1}{\lambda_{n}}$. It immediately follows

$$
\begin{gathered}
V_{n}^{(\alpha, \beta}\left(e_{0} ; \lambda_{n}\right)(x)=U_{n, 0}^{(\alpha, \beta)}\left(x ; \lambda_{n}\right)=1, \\
V_{n}^{(\alpha, \beta)}\left(e_{1} ; \lambda_{n}\right)(x)=U_{n, 1}^{(\alpha, \beta)}\left(x ; \lambda_{n}\right)=\frac{1}{1+\lambda_{n} \beta} x+\frac{\lambda_{n}+\lambda_{n} \alpha}{1+\lambda_{n} \beta}, \\
V_{n}^{(\alpha, \beta)}\left(e_{2} ; \lambda_{n}\right)(x)=U_{n, 2}^{(\alpha, \beta)}\left(x ; \lambda_{n}\right)=\frac{1+\lambda_{n}}{\left(1+\lambda_{n} \beta\right)^{2}} x^{2}+\frac{4 \lambda_{n}+2 \lambda_{n} \alpha}{\left(1+\lambda_{n} \beta\right)^{2}} x+\frac{\lambda_{n}^{2} \alpha^{2}+2 \lambda_{n}^{2} \alpha+2 \lambda_{n}^{2}}{\left(1+\lambda_{n} \beta\right)^{2}} .
\end{gathered}
$$

(ii) We have $V_{n}^{(\alpha, \beta)}\left((t-x)^{2} ; \lambda_{n}\right)(x)$

$$
\begin{gathered}
=V_{n}^{(\alpha, \beta)}\left(e_{2} ; \lambda_{n}\right)(x)-2 x V_{n}^{(\alpha, \beta)}\left(e_{1} ; \lambda_{n}\right)(x)+x^{2} V_{n}^{(\alpha, \beta)}\left(e_{0} ; \lambda_{n}\right)(x) \\
=\frac{1+\lambda_{n}}{\left(1+\lambda_{n} \beta\right)^{2}} x^{2}+\frac{4 \lambda_{n}+2 \lambda_{n} \alpha}{\left(1+\lambda_{n} \beta\right)^{2}} x+\frac{\lambda_{n}^{2} \alpha^{2}+2 \lambda_{n}^{2} \alpha+2 \lambda_{n}^{2}}{\left(1+\lambda_{n} \beta\right)^{2}} \\
-2 x\left(\frac{1}{1+\lambda_{n} \beta} x+\frac{\lambda_{n}+\lambda_{n} \alpha}{1+\lambda_{n} \beta}\right)+x^{2} \\
=\frac{\lambda_{n}+\lambda_{n}^{2} \beta^{2}}{\left(1+\lambda_{n} \beta\right)^{2}} x^{2}+\frac{2 \lambda_{n}-2 \lambda_{n}^{2} \beta-2 \lambda_{n}^{2} \alpha \beta}{\left(1+\lambda_{n} \beta\right)^{2}} x+\frac{\lambda_{n}^{2} \alpha^{2}+2 \lambda_{n}^{2} \alpha+2 \lambda_{n}^{2}}{\left(1+\lambda_{n} \beta\right)^{2}},
\end{gathered}
$$

which proves the lemma. 
The main result of this section is the following.

Theorem 5.2. Let $0 \leq \alpha \leq \beta, \lambda_{n} \searrow 0$ as fast we want and suppose that $f:[0,+\infty) \rightarrow$ $\mathbb{R}$ is uniformly continuous on $[0,+\infty)$. For all $x \in[0,+\infty)$ and $n \in \mathbb{N}$, we have

$$
\left|V_{n}^{(\alpha, \beta)}\left(f ; \lambda_{n}\right)(x)-f(x)\right| \leq 2 \omega_{1}\left(f ; \sqrt{\lambda_{n}} \cdot \sqrt{F_{n}^{(\alpha, \beta)}(x)}\right),
$$

where

$$
F_{n}^{(\alpha, \beta)}(x)=\frac{1+\lambda_{n} \beta^{2}}{\left(1+\lambda_{n} \beta\right)^{2}} x^{2}+\frac{2-2 \lambda_{n} \beta-2 \lambda_{n} \alpha \beta}{\left(1+\lambda_{n} \beta\right)^{2}} x+\frac{\lambda_{n} \alpha^{2}+2 \lambda_{n} \alpha+2 \lambda_{n}}{\left(1+\lambda_{n} \beta\right)^{2}} .
$$

Proof. Reasoning exactly as in the proof of Theorem 2.2, we can write

$$
\left|V_{n}^{(\alpha, \beta)}\left(f ; \lambda_{n}\right)(x)-f(x)\right| \leq\left(1+\delta^{-1} \sqrt{V_{n}^{(\alpha, \beta)}\left(\varphi_{x}^{2} ; \lambda_{n}\right)(x)}\right) \omega_{1}(f ; \delta) .
$$

Choosing here $\delta=\sqrt{V_{n}^{(\alpha, \beta)}\left(\varphi_{x}^{2} ; \lambda_{n}\right)(x)}$ and using Lemma 5.1, (ii), we obtain

$$
\left|S_{n}^{(\alpha, \beta)}\left(f ; \lambda_{n}\right)(x)-f(x)\right| \leq 2 \omega_{1}\left(f ; \sqrt{\lambda_{n}} \cdot \sqrt{F_{n}^{(\alpha, \beta)}(x)}\right),
$$

which proves the theorem.

As an immediate consequence of Theorem 5.2 we get the following.

Corollary 5.3. Let $0 \leq \alpha \leq \beta, \lambda_{n} \searrow 0$ as fast we want and suppose that $f$ is a Lipschitz function, that is there exists $M>0$ such that $|f(x)-f(y)| \leq M|x-y|$, for all $x, y \in[0, \infty)$. Then, for all $x \in[0,+\infty)$ and $n \in \mathbb{N}$ we have

$$
\left|V_{n}^{(\alpha, \beta)}\left(f ; \lambda_{n}\right)(x)-f(x)\right| \leq 2 M \sqrt{\lambda_{n}} \cdot \sqrt{F_{n}^{(\alpha, \beta)}(x)} .
$$

Proof. Since by hypothesis $f$ is a Lipschitz function, we easily get $\omega_{1}(f ; \delta) \leq M \delta$, for all $\delta>0$. Choosing now $\delta=\sqrt{\lambda_{n}} \cdot \sqrt{F_{n}^{(\alpha, \beta)}(x)}$ and applying Theorem 5.2 we get the desired estimate.

Remarks. 1) All the Remarks 1)-3) made at the end of Section 2 remain valid for the generalized Baskakov-Szász-Durrmeyer-Stancu, $V_{n}^{(\alpha, \beta)}\left(f ; \lambda_{n}\right)$, operators too.

2) Note that in Theorems 2.2, 3.2, 4.2 and 5.2 , for any $\delta>0$ and $f:[0,+\infty) \rightarrow \mathbb{R}$ uniformly continuous, the modulus of continuity $\omega_{1}(f ; \delta)$ is finite. For the reader's convenience, we present below the proof. Indeed, for a fixed $\varepsilon_{0}$, from the definition of the uniform continuity of $f$, there exists a $\delta_{0}>0$, such that $|f(x)-f(y)|<\varepsilon_{0}$, for all $x, y \in[0,+\infty)$ with $|x-y| \leq \delta_{0}$. Passing here to supremum after these $x, y$, it immediately follows that $\omega_{1}\left(f ; \delta_{0}\right) \leq \varepsilon_{0}<+\infty$. Let now $\delta>\delta_{0}$ be arbitrary. Evidently that there exists a sufficiently large $p \in \mathbb{N}$, such that $\delta \leq p \cdot \delta_{0}$. Using now the monotonicity and the subadditivity of $\omega_{1}(f ; \delta)$ as function of $\delta$, we get

$$
\omega_{1}(f ; \delta) \leq \omega_{1}\left(f ; p \delta_{0}\right) \leq p \cdot \omega_{1}\left(f ; \delta_{0}\right)<+\infty .
$$

Finally, we may conclude that the approximation results obtained for all the operators in this paper are of a definitive character, i.e. they furnish arbitrary good orders of approximation. It is also worth noting that the method in this paper does not work for the positive and linear operators expressed by finite sums (like Bernstein polynomials, Kantorovich polynomials, etc). 


\section{References}

[1] Agratini, O., Approximation by Linear Operators (Romanian), University Press, BabeşBolyai University, Cluj-Napoca, 2000.

[2] Baskakov, V.A., An example of a sequence of linear positive operators in the space of continuous functions (Russian), Dokl. Akad. Nauk SSSR, 113(1957), 249-251.

[3] Boehme, T.K., Bruckner, A.M., Functions with convex means, Pacific J. Math., 14(1964), 1137-1149.

[4] Dieudonné, J., Éléments dAnalyse; 1. Fondements de l'Analyse Moderne, Gauthiers Villars, Paris, 1968.

[5] Djebali, S., Uniform continuity and growth of real continuous functions, Int. J. Math. Education in Science and Technology, 32(2001), no. 5, 677-689.

[6] Gal, S.G., Approximation with an arbitrary order by generalized Szász-Mirakjan operators, Stud. Univ. Babeş-Bolyai Math., 59(2014), no. 1, 77-81.

[7] Gal S.G., Gupta, V., Approximation by complex Szász-Durrmeyer operators in compact disks, Acta Math. Scientia, 34B(2014), no. 4, 1157-1165.

[8] Gal, S.G., Gupta, V., Approximation by complex Szász-Mirakjan-Stancu-Durrmeyer operators in compact disks under exponential growth, Filomat, 29(2015), no. 5, 1127-1136.

[9] Gal, S.G., Opriş, D.B., Approximation with an arbitrary order by modified Baskakov type operators, Appl. Math. Comp., 265(2015), 329-332.

[10] Gal, S.G., Opriş, D.B., Approximation of analytic functions with an arbitrary order by generalized Baskakov-Faber operators in compact sets, Complex Anal. Oper. Theory, 10(2016), no. 2, 369-377.

[11] Gupta, V., Complex Baskakov-Szász operators in compact semi-disks, Lobachevskii J. Math., 35(2014), no. 2, 65-73.

[12] Gupta, V., Overconvergence of complex Baskakov-Szász-Stancu operators, Mediterr. J. Math., 12(2015), no. 2, 455-470.

[13] Mazhar, S.M., Totik V., Approximation by modified Szász operators, Acta Sci. Math., 49(1985), 257-269.

[14] Shisha, O., Mond, B., The degree of convergence of linear positive operators, Proc. Nat. Acad. Sci. U.S.A., 60(1968), 1196-1200.

[15] Totik, V., Approximation by Szász-Mirakjan-Kantorovich operators in $L^{p}(p>1)$ (Russian), Analysis Math., 9(1983), no. 2, 147-167.

[16] Walczak, Z., On approximation by modified Szász-Mirakjan operators, Glasnik Mat., 37(2002), no. 2, 303-319.

Sorin Trifa

Faculty of Mathematics and Computer Science

Babeş-Bolyai University

Cluj-Napoca, Romania

e-mail: sorin.trifa@yahoo.com 